
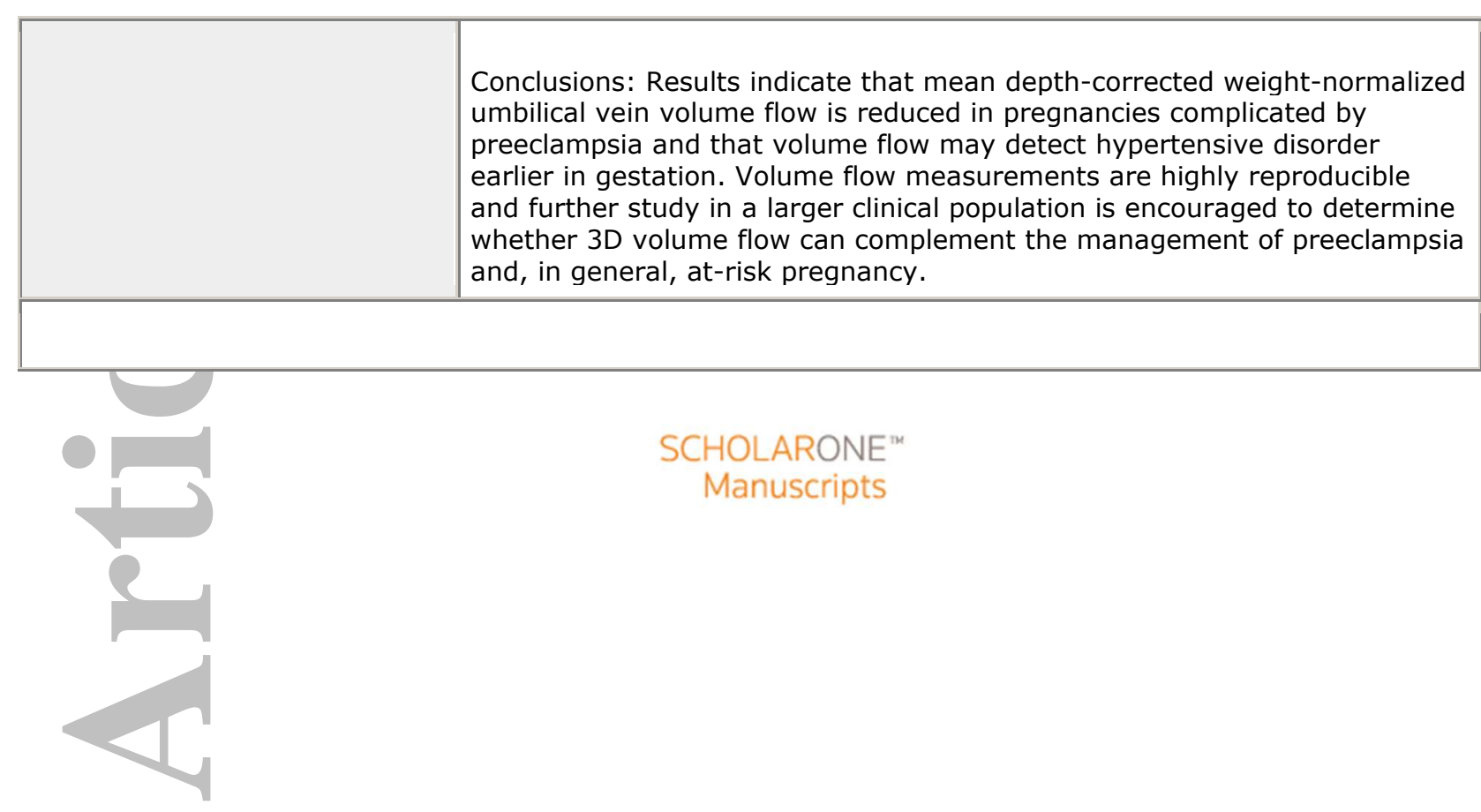

SCHOLARONE ${ }^{m}$

Manuscripts

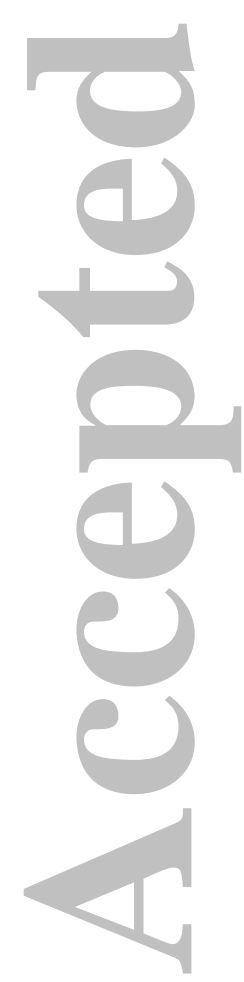

This is the author manuscript accepted for publication and has undergone full peer review but has not been through the copyediting, typesetting, pagination and proofreading process, which may lead to differences between this version and the Version record. Please cite this article as doi:10.1002/ jum.14507. 


\section{Evaluation of Umbilical Vein Blood Volume Flow in Preeclampsia by Angle-Independent 3D Sonography}

Stephen Z Pinter PhD*, Oliver D Kripfgans PhD*, Marjorie C Treadwell MD†, Anna W Kneitel MD†, J Brian Fowlkes PhD*, Jonathan M Rubin MD PhD*

*Department of Radiology, University of Michigan, Ann Arbor, MI, USA

†Department of Obstetrics and Gynecology, University of Michigan, Ann Arbor, MI, USA

Corresponding author: Oliver D Kripfgans (oliver.kripfgans@umich.edu, (734) 647-0852)

\section{Author who is to check proofs:}

Stephen Z Pinter (pinters@umich.edu)

University of Michigan, Department of Radiology

Medical Science Building I, Rm 3232

1301 Catherine Street

Ann Arbor, MI 48109-5667

Tel: (734) 615-0152, Fax: (734) 764-8541

Manuscript category: Original Research

Short title: Umbilical Flow in Preeclampsia using 3D Sonography 


\section{Evaluation of Umbilical Vein Blood Volume Flow in Preeclampsia by Angle-Independent 3D Sonography}

Abstract

Objectives Investigate the association between umbilical vein blood volume flow and the condition of preeclampsia in an at-risk maternal patient cohort. Umbilical vein volume flow is quantified using a three-dimensional (3D) sonographic technique that overcomes several limitations of standard sonographic flow measurement methods.

Methods A total of 35 patients, each with a singleton pregnancy, were recruited to provide 5 preeclampsia subjects (gestation: 29.7-34.3 weeks), derived as a subset from a 26-patient atrisk group, and 9 normal subjects (gestation: 25.9-34.7 weeks). An ultrasound system equipped with a 2.0-8.0-MHz probe was used to acquire multi-volume 3D color flow and power mode data sets in order to compute mean umbilical vein volume flow in normal and preeclampsia subjects.

Results Comparisons between normal and preeclampsia subjects showed weight-normalized flow with a moderately high separation between groups $(P=0.11)$ and depth-corrected weightnormalized flow with a statistically significant difference between groups $(P=0.035)$. Umbilical vein volume flow measurements were highly reproducible in the mean estimate with an intrasubject relative standard error (RSE) of $12.1 \pm 5.9 \%$ and an intra-measurement RSE of $5.6 \pm$ $1.9 \%$. In patients that developed pregnancy-induced hypertension (PIH) or severe pregnancyinduced hypertension (sPIH), umbilical vein volume flow suggested gestational hypertensive disorder prior to clinical diagnosis.

Conclusions Results indicate that mean depth-corrected weight-normalized umbilical vein volume flow is reduced in pregnancies complicated by preeclampsia and that volume flow may 
detect hypertensive disorder earlier in gestation. Volume flow measurements are highly reproducible and further study in a larger clinical population is encouraged to determine whether 3D volume flow can complement the management of preeclampsia and, in general, at-risk pregnancy.

Key Words volume flow; umbilical vein blood flow; high-risk pregnancy; preeclampsia; color flow; power mode; c-surface imaging; vascular sonography

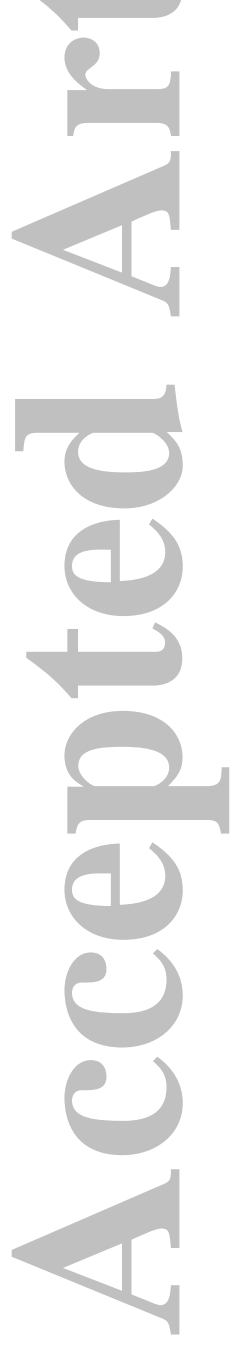


Introduction

Umbilical cord blood flow is critical to fetal development and its significance cannot be overstated. An editorial in Ultrasound in Obstetrics \& Gynecology by Ferrazzi, entitled "Measurement of venous blood flow in the human fetus: a dream comes true, but now for some standardization", tells it all. ${ }^{1}$ Umbilical cord flow is among the highly desired parameters for monitoring fetal well being since cord flow is directly related to placental volume flow, a parameter considered as important in the fetus as cardiac output and lung perfusion in adults. ${ }^{2}$ An accurate, reliable, and rapid method for measuring umbilical cord volume flow would help the obstetric provider identify fetuses with abnormal flow, such as decreased flow in intrauterine growth restriction (IUGR), ${ }^{3}$ and potentially those at increased risk for an adverse outcome.

Currently, there are well-known problems with standard sonographic volume flow measurement techniques in terms of the required assumptions: cylindrically symmetric flow velocity profile, circular vessel cross section, and a mean velocity estimate that requires angle correction. ${ }^{4}$ Each supposition is tenuous in umbilical cords where vessel paths are helical. Helical geometry generally invalidates the assumption of a cylindrically symmetric flow profile. ${ }^{5}$ Angle correction is difficult given the constant change in vessel geometry. In addition, the umbilical vein, which some consider the preferred vessel for analysis, ${ }^{6,7}$ is often not circular in cross section. In general, standard volume flow methods are rarely used in clinical practice. Despite these limitations, many investigators have evaluated umbilical cord blood flow in fetal populations and showed that flow is relevant when assessing fetal well being and placental function. ${ }^{2,3,8-15}$

Our group has been developing and evaluating a three-dimensional (3D) sonographic method for measuring blood volume flow that overcomes nearly all limitations of standard techniques and is independent of flow velocity profile, vessel geometry, and vessel angle, as long as there 
is detectable color signal. ${ }^{16-19}$ Using this $3 \mathrm{D}$ approach, umbilical vein blood volume flow is quantified in a small cohort of patients, and measurements are compared between at-risk pregnancies diagnosed with preeclampsia and normal pregnancies. Gestational timelines for preeclampsia subjects are used to determine whether 3D volume flow can indicate gestational hypertensive disorder prior to clinical diagnosis. In addition, umbilical vein volume flow measurements are evaluated in terms of intra-subject and intra-measurement variability. Overall, if the $3 D$ method is viable, the approach could simplify umbilical cord volume flow measurement and may provide a new and valuable clinical parameter to assess fetal well being.

\section{Materials and Methods}

\section{Clinical Cohort}

A total of 35 patients, each with a singleton pregnancy (gestation range: 22.0-37.0 weeks), were recruited from the University of Michigan Medical Center Maternal Fetal Medicine Clinics and consecutively enrolled in this prospective study. Each patient provided fully informed written consent to an Institutional Review Board approved protocol involving transcutaneous measurement of fetal blood volume flow with sonography. The study is HIPAA compliant.

Patients were recruited from a population known to be at an increased risk of preeclampsia (risk factors included: hypertension, obesity, diabetes, advanced maternal age). A preeclampsia group was derived as a subset from the at-risk group and consisted of patients who either had preeclampsia at the time of enrollment or who went on to develop preeclampsia. An additional subset of patients without risk factors for preeclampsia or growth abnormalities was also recruited. Overall, the 35-patient cohort can be classified into three groups: 21 at-risk subjects (gestation range: 22.0-37.0 weeks), 5 preeclampsia subjects (gestation range: 29.7-34.3 weeks), and 9 normal subjects (gestation range: 25.9-34.7 weeks). 
In utero fetal weight at the time of ultrasound scan was estimated based on a spline interpolation of 50 th percentile weight data. ${ }^{20}$ Patients were followed to full term and final outcomes were verified in medical records.

\section{Sonographic Data Acquisition}

A LOGIQ E9 ultrasound system (GE Healthcare, Milwaukee, WI, USA) equipped with a 2.0-8.0$\mathrm{MHz}$ bandwidth convex array transducer (RAB6-D) was used to acquire multi-volume 3D color flow and power mode data sets. A custom imaging preset with scanner parameters optimized for acquiring volume flow data was stored on the system and used in all scans. Each multivolume data set consisted of 30 (nominal) individual volumes. An individual volume acquisition would occasionally be discarded due to fetal or maternal movement during the sweep, but volumes would continue to be acquired sequentially until the multi-volume data set was complete. Approximately 5-10 minutes were required to collect a multi-volume data set.

Two examiners highly skilled in obstetrical ultrasound performed the imaging (M.C.T.; J.M.R.). Each individual imaging study was performed by one examiner. Examiners were completely blinded to the volume flow measurement results since calculations are performed offline.

Umbilical vessels of a free cord loop were visualized in the lateral-elevational imaging plane (csurface) by adjusting the position and tilt angle of the probe until the c-surface fully intersected the vein and both arteries with sufficient margin. For the duration of each multi-volume scan, the probe was held primarily in said position unless there was fetal or maternal movement; the probe would then be repositioned to maintain vessels in the c-surface. The color flow axial focus was aligned directly with, or as near as possible to, the c-surface depth. Umbilical cord imaging depths ranged from 3.3 to $11.0 \mathrm{~cm}$. Glancing exposure to the fetus was largely avoided. 
For each patient, mean umbilical vein volume flow was assessed at three different free loop positions along the length of the umbilical cord. In five patients, volume flow was assessed at only two positions, and in one patient, volume flow was assessed at only one position, for reasons that include: poor color flow image quality, patient having to leave the study early, and lack of study time due to a highly active fetus (i.e., substantial cord movement).

Volume Flow Analysis

Multi-volume 3D data sets were exported as duplex mode DICOMs, along with the corresponding scanner acquisition settings, for offline processing and prospective analysis. Volume flow for each multi-volume data set was computed (S.Z.P.) using custom algorithms implemented in MATLAB (MathWorks, Natick, MA, USA) through a user-specified c-surface by the method of surface integration of color flow-measured velocity vectors. ${ }^{21,22}$ Power mode data was used to correct for the partial volume effect. ${ }^{16-19}$

Mean volume flow in the umbilical vein is computed by the summation of flow in all voxels that intersect the vessel. Volume flow in each voxel is computed by $Q=v \times(A \times w)$, where $v$ is mean local color flow-measured velocity, $A$ is area of the local surface element, and $w(0 \leq w \leq$ 1 ) is a local weighting factor computed in reference to the power in voxels that contain $100 \%$ blood and indicates the voxel area fraction that intersects the vessel. The above equation is only applicable when velocity is normal to the c-surface area element, a condition satisfied by the probe's scan geometry. Prior articles ${ }^{16-19}$ provide further review on power-weighted surface integration of color flow-measured velocity vectors.

Umbilical vein volume flow was computed on an image volume-by-volume basis for each multivolume data set and overall flow was recorded as the mean of individual flow estimates. Image 
volume-by-volume flow computation is necessary for the umbilical application because it permits movement of the cord within the imaging volume of interest between subsequent sweeps by the $3 \mathrm{D}$ transducer. In order to compute flow only in the umbilical vein, the vessel was isolated from adjacent arteries by directional criteria and from local color flow artifact by a broad manual segmentation (S.Z.P.) since partial volume correction is ultimately used to determine the vessel's boundary.

\section{Results}

Figure 1 shows a representative color flow image obtained from a single 3D sweep in an at-risk subject of umbilical vessels in a free cord loop visualized in the axial-lateral and lateralelevational (c-surface) imaging planes. Umbilical vein and arteries fully intersect the c-surface with adequate margin. A cross-sectional orientation of the cord in the c-surface (as shown in Figure 1) is the required view for $3 D$ flow measurement and was achieved with all scans performed in this study.

Figure 2 shows the gestational age dependence of umbilical vein volume flow measurement. Mean absolute flow increases with gestational age, and there appears to be a lack of flow separation between normal and preeclampsia subjects; although, several preeclampsia data points are lower in comparison. Note that these flow measurements are not weight normalized.

Figure 3 demonstrates the depth dependence of umbilical vein volume flow measurement. Linear regression analyses show that mean absolute flow increases with vessel depth for both normal and preeclampsia subjects. Figure 3 depicts the linear regression only for the normal group. Details on the linear regressions for both the normal and preeclampsia groups are 
provided in the caption. A comparison of linear regressions indicates that the difference between slopes for the two groups is not significant $(P=0.92)$.

A variability analysis of umbilical vein volume flow is given in Table 1 , where intra-subject variability is based on a subject's free loop flow estimates and intra-measurement variability is based on the multi-volume data set acquired at a free loop position. Multi-volume data sets consisted of $28.3 \pm 3.3$ (mean $\pm \mathrm{SD}$ ) individual volumes. Relative standard deviation (RSD), i.e., coefficient of variation (CV), and relative standard error (RSE) values reported in Table 1 are computed using absolute flow estimates and are averaged over the entire cohort of subjects.

Figure 4 provides comparisons between mean umbilical vein volume flow in normal and preeclampsia subjects based on the following metrics: absolute flow, depth-corrected flow, weight-normalized flow, and depth-corrected weight-normalized flow. Depth corrected refers to an adjustment based on the linear regression slope of normal subjects $(22.73 \mathrm{~mL} / \mathrm{min} / \mathrm{cm}$, Figure 3) that accounts for the depth dependence of elevational beam width when away from the elevational focus. Absolute flow (Figure 4(a)) and depth-corrected flow (Figure 4(b)) show moderate separation between groups $(P=0.58, P=0.41)$. Weight-normalized flow (Figure 4(c)) shows a moderately high separation between groups $(P=0.11)$. Depth-corrected weightnormalized flow (Figure 4(d)) shows a statistically significant difference between groups $(P=$ $0.035)$.

Table 2 shows the ultrasound volume flow scan, gestational diagnosis, and delivery timelines for preeclampsia subjects. Note that for patients with pregnancy-induced hypertension $(\mathrm{PIH})$ or severe pregnancy-induced hypertension (sPIH), the clinical diagnosis was made on delivery. 


\section{Discussion}

Standard sonographic evaluation of umbilical cord blood flow is not uncommon. Umbilical artery waveform analyses use surrogate flow parameters, e.g., systolic/diastolic (S/D) ratios, but such parameters fail to directly reflect placental-fetal blood flow, and changes often occur too late for use as a screening tool in low-risk pregnancies. ${ }^{12,23}$ Further, recent work has shown that umbilical artery velocity indices are generally insensitive to distinguish many small for gestational age fetuses from those with IUGR. ${ }^{24}$ Such indices are typically derived from flow velocity estimates that are acquired using standard pulsed wave techniques and their values are only related to umbilical cord flow, thus, the indices fail to indicate true flow. Also, the indices are often difficult to interpret and can vary depending on measurement location in the umbilical cord. $^{25}$

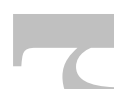

Volume flow estimation is a considerably different approach. Volume flow changes in the umbilical vein have been shown to occur before umbilical artery flow indices become abnormal. $^{10}$ Volume flow also has the attractive property of being a parameter that directly reflects placental blood volume flow, the pathophysiologic parameter of interest. ${ }^{2}$ Further, due to conservation of mass, volume flow should be equivalent everywhere along the length of the cord and the average flow in umbilical arteries must equal that in the vein. Therefore, volume flow measurements should be independent of the position where they are acquired along the cord.

Fetal volume flow has been a target of serious investigation since the early 1980s; however, the measure has had continual technical problems such as large measurement variability and the requirement for significant operator skill. ${ }^{1}$ In fact, it has been explicitly stated that volume flow measures are presently too inaccurate and too difficult to reproduce to be clinically relevant. ${ }^{7}$ 
Nevertheless, placental-fetal blood flow is considered such a valued parameter that recent studies have continued to focus on umbilical vein volume flow despite the limitations. ${ }^{6,7}$ One group of observers indicated that umbilical vein volume flow estimation is tractable and can be performed by a non-expert operator. ${ }^{1}$ Although, the ultimate reality seems to be that volume flow estimation using standard pulsed wave, as performed presently, is highly variable and difficult to perform. ${ }^{1,7,12,24}$

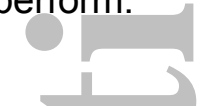

Our approach for blood volume flow measurement employs 3D sonography, which overcomes several technical limitations of standard flow methods. ${ }^{16-18}$ In theory, this $3 \mathrm{D}$ method is independent of flow velocity profile, vessel geometry, and vessel angle, as long as there is detectable color signal. Our intention is for blood volume flow estimation in the umbilical vein to be as straightforward as standard color flow imaging. In this study, the 3D method was used to measure umbilical vein volume flow in a small cohort of patients, and results were compared between at-risk pregnancies diagnosed with preeclampsia and normal pregnancies.

Preeclampsia is a condition defined by maternal hypertension and proteinuria, and can evolve into eclampsia when seizures develop. ${ }^{26}$ Preeclampsia is one of the leading causes of maternal and fetal mortality, although the cause of the condition is still under investigation. ${ }^{26} \mathrm{~A}$ general consensus is that the cause of preeclampsia is placenta based; however, beyond that there are multiple theories and risk factors that include: previous history of preeclampsia, nulliparity, multiple pregnancy, high BMI, more than 10 years since prior pregnancy, and hypertension. ${ }^{27}$ Other associations include low dietary calcium intake and undernutrition. ${ }^{28-30}$ Multiple genetic factors have also been associated with preeclampsia. ${ }^{26}$ If the onset of preeclampsia could potentially be predicted based on decreased umbilical cord volume flow, this may provide an avenue for investigating and understanding this serious and mysterious condition. In this regard, this study detected a statistically significant difference in mean depth-corrected weight- 
normalized umbilical vein blood volume flow between normal pregnancies and those complicated by preeclampsia $(P=0.035)$.

Based on the study timelines for preeclampsia subjects (Table 2), umbilical vein volume flow suggested gestational hypertensive disorder at, or prior to, the clinical diagnosis in four out of five patients $(13,16,19,22)$. In patients that developed $\mathrm{PIH}$ or $\operatorname{sPIH}(13,16,18,19)$, volume flow suggested disorder prior to the clinical diagnosis in all four cases. An earlier scheduled ultrasound volume flow scan could potentially improve detection time and lead to earlier intervention. Furthermore, although the analysis in Table 1 showed an elevated estimate variability (RSD), RSE showed that estimate precision for mean volume flow approached reasonable values when acquiring a practical number of repeated samples. Implementation of the 3D volume flow method on a 2D array transducer could provide repeated samples within seconds, which would significantly reduce the time required to acquire a multi-volume data set and to sample multiple free loop positions.

Preliminary results presented here encourage further investigation, but there are some limitations with the current study. First, the size of the recruited population is limited, and therefore these results should be confirmed in a larger population. A sole objective diagnostic judgment is not recommended based on the reported results given the small sample size. Currently, this proposed application of $3 D$ volume flow in preeclampsia should be regarded a complementary or additive diagnostic criterion until the conclusions are validated in a larger study. Although preeclampsia was the condition under investigation, in such a small sample size it is possible that the detected difference in umbilical vein volume flow is suggestive more generally of hypertensive disorder. In a larger study, one could investigate umbilical vein volume flow across a spectrum of hypertensive disorders and determine to which the method is most sensitive and specific. 
Second, multi-volume data sets were collected with a mechanically swept 3D ultrasound probe, which requires several seconds to sweep a volume, i.e., to acquire a c-surface, through the umbilical cord. If the fetus moves the cord during this time-consuming step, the flow data, and thus the measurement, is invalid and additional volume scans are required. Third, the mechanically swept 3D probe has a fixed elevational focus where the c-surface is best defined. Outside this focal depth, the beam broadens, and a $100 \%$ reference blood measurement for partial volume correction may not exist. We had to compensate for imaging depth in flow estimates likely because of this fixed elevational focus. This effect should be reduced with a 2D array transducer, where the elevational focus can be controlled electronically to provide a more tightly focused elevational beam.

Three-dimensional volume flow is currently under development by the Quantitative Imaging Biomarkers Alliance (QIBA) in an effort to evaluate measurement variability across users and systems. Based on these QIBA outcomes, we will be able to determine the degree of variability expected between users for 3D volume flow measurements in the umbilical vein.

In conclusion, using a 3D sonographic method to measure umbilical vein blood volume flow in a small cohort of patients, a difference in flow was detected between normal pregnancies and those complicated by preeclampsia. If this result remains consistent with further investigation, the proposed $3 \mathrm{D}$ method could provide a quantitative and robust flow metric to help predict pregnancies that will develop hypertension and proteinuria before signs and symptoms arise. 


\section{Acknowledgments}

Funding support (partial) was provided by the American Institute of Ultrasound in Medicine (AIUM) Endowment for Education and Research (EER). Equipment support was provided by GE Healthcare (ultrasound).
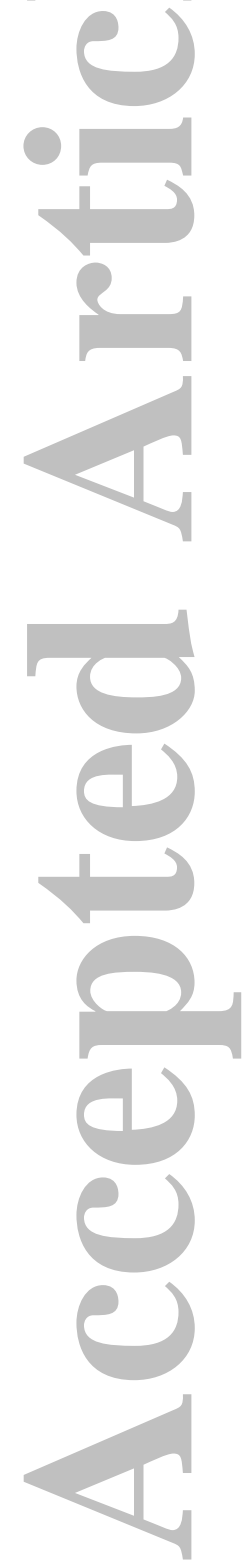


\section{References}

1. Ferrazzi E. Measurement of venous blood flow in the human fetus: a dream comes true, but now for some standardization. Ultrasound Obstet Gynecol. 2001;18:1-4.

2. Tchirikov M, Rybadowski C, Huneke B, Schoder V, Schroder HJ. Umbilical vein blood volume flow rate and umbilical artery pulsatility as 'venous-arterial index' in the prediction of neonatal compromise. Ultrasound Obstet Gynecol. 2002;20:580-5.

3. Ferrazzi E, Rigano S, Bozzo M, Giovannini N, Galan H, Battaglia FC. Umbilical vein blood flow in growth-restricted fetuses. Ultrasound Obstet Gynecol. 2000;16:432-8.

4. Gill R. Measurement of blood flow by ultrasound: accuracy and sources of error. Ultrasound in Medicine and Biology. 1985;11:625-41.

5. Berger SA, Talbot L, Yao L-S. Flow in curved pipes. Ann Rev Fluid Mech. 1983;15:461512.

6. Figueras F, Fernandez S, Hernandez-Andrade E, Gratacos E. Umbilical venous blood flow measurement: accuracy and reproducibility. Ultrasound Obstet Gynecol. 2008;32(4):58791.

7. Flo K, Wilsgaard T, Acharya G. Agreement between umbilical vein volume blood flow measurements obtained at the intra-abdominal portion and free loop of the umbilical cord. Ultrasound Obstet Gynecol. 2009;34(2):171-6.

8. Lees C, Albaiges G, Deane C, Parra M, Nicolaides KH. Assessment of umbilical arterial and venous flow using color Doppler. Ultrasound Obstet Gynecol. 1999;14:250-5.

9. Bellotti M, Pennati G, De Gasperi C, Bozzo M, Battaglia FC, Ferrazzi E. Simultaneous measurements of umbilical venous, fetal hepatic, and ductus venosus blood flow in growthrestricted human fetuses. Am J Obstet Gynecol. 2004;190(5):1347-58. 
0. Rigano S, Bozzo M, Ferrazzi E, Bellotti M, Battaglia FC, Galan HL. Early and persistent reduction in umbilical vein blood flow in the growth-restricted fetus: a longitudinal study. Am J Obstet Gynecol. 2001;185:834-8.

1. Najafzadeh A, Dickinson JE. Umbilical venous blood flow and its measurement in the human fetus. J Clin Ultrasound. 2012;40(8):502-11.

2. Acharya G, Wilsgaard T, Bernsten GKR, Maltau JM, Kiserud T. Doppler-derived umbilical artery absolute velocities and their relationship to fetoplacental volume blood flow: a longitudinal study. Ultrasound Obstet Gynecol. 2005;25:444-53.

3. Boito S, Struijk PC, Ursem NT, Stijnen T, Wladimiroff JW. Umbilical venous volume flow in the normally developing and growth-restricted fetus. Ultrasound Obstet Gynecol. 2002;19:344-9.

4. Boito SM, Ursem NT, Struijk PC, Stijnen T, Wladimiroff JW. Umbilical venous volume flow and fetal behavioral states in the normally developing fetus. Ultrasound Obstet Gynecol. $2004 ; 23(2): 138-42$.

5. Ferrazzi E, Bellotti M, Galan H, Pennati G, Bozzo M, Rigano S, Battaglia FC. Doppler investigation in intrauterine growth restriction--from qualitative indices to flow measurements: a review of the experience of a collaborative group. Annals of the New York Academy of Sciences. 2001;943:316-25.

6. Kripfgans OD, Rubin JM, Hall AL, Gordon MB, Fowlkes JB. Measurement of volumetric flow. J Ultrasound Med. 2006;25:1305-11.

7. Richards MS, Kripfgans OD, Rubin JM, Hall AL, Fowlkes JB. Mean volume flow estimation in pulsatile flow conditions. Ultrasound Med Biol. 2009;35(11):1880-91.

8. Pinter SZ, Rubin JM, Kripfgans OD, Treadwell MC, Romero VC, Richards MS, Zhang M, Hall AL, Fowlkes JB. Three-dimensional sonographic measurement of blood volume flow in the umbilical cord. J Ultrasound Med. 2012;31(12):1927-34. 
9. Pinter SZ, Rubin JM, Kripfgans OD, Novelli PM, Vargas-Vila M, Hall AL, Fowlkes JB. Volumetric blood flow in transjugular intrahepatic portosystemic shunt revision using 3dimensional Doppler sonography. J Ultrasound Med. 2015;34(2):257-66.

0. Hadlock FP, Harrist RB, Martinez-Poyer J. In utero analysis of fetal growth: a sonographic weight standard. Radiology. 1991;181(1):129-33.

1. Moser U, Vieli A, Schumacher P, Pinter P, Basler S, Anliker M. [A Doppler ultrasound device for determining blood volume flow]. Ultraschall Med. 1992;13(2):77-9.

2. Sun $Y$, Ask P, Janerot-Sjoberg B, Eidenvall L, Loyd D, Wranne B. Estimation of volume flow rate by surface integration of velocity vectors from color Doppler images. J Am Soc Echocardiogr. 1995;8(6):904-14.

3. Newnham JP, Patterson LL, James IR, Diepeveen DA, Reid SE. An evaluation of the efficacy of Doppler flow velocity waveform analysis as a screening test in pregnancy. Am J Obstet Gynecol. 1990;162:403-10.

4. Figueras F, Eixarch E, Gratacos E, Gardosi J. Predictiveness of antenatal umbilical artery Doppler for adverse pregnancy outcome in small-for-gestational-age babies according to customised birthweight centiles: population-based study. BJOG. 2008;115(5):590-4.

5. Mehalek KE, Rosenberg J, Berkowitz GS, Chitkara U, Berkowitz RL. Umbilical and uterine artery flow velocity waveforms. Effect of the sampling site on Doppler ratios. J Ultrasound Med. 1989;8(4):171-6.

6. Noris M, Perico N, Remuzzi G. Mechanisms of disease: Pre-eclampsia. Nature clinical practice Nephrology. 2005;1(2):98-114; quiz 20.

7. Duckitt K, Harrington D. Risk factors for pre-eclampsia at antenatal booking: systematic review of controlled studies. BMJ. 2005;330(7491):565.

8. Atallah AN, Hofmeyr GJ, Duley L. Calcium supplementation during pregnancy for preventing hypertensive disorders and related problems. Cochrane Database Syst Rev. 2002(1):CD001059. 
9. Hofmeyr GJ, Roodt A, Atallah AN, Duley L. Calcium supplementation to prevent preeclampsia--a systematic review. South African medical journal = Suid-Afrikaanse tydskrif vir geneeskunde. 2003;93(3):224-8.

0. Villar J, Belizan JM. Same nutrient, different hypotheses: disparities in trials of calcium supplementation during pregnancy. The American journal of clinical nutrition. 2000;71(5 Suppl):1375S-9S.
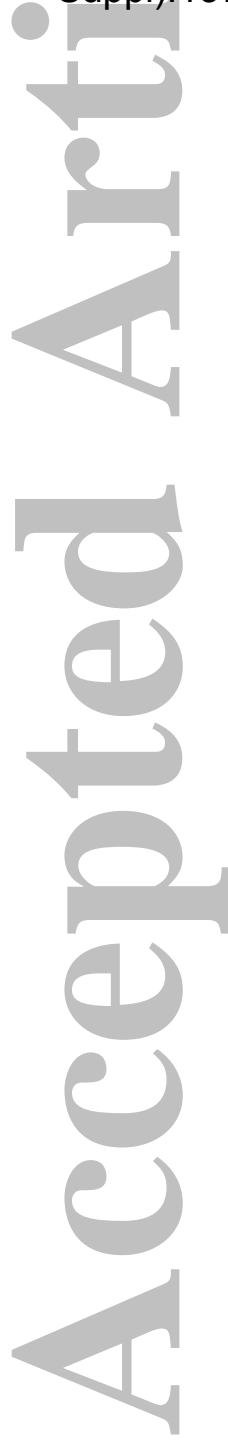
Table 1. Volume Flow Estimate Variability (all subjects, absolute flow)

\begin{tabular}{|l|c|}
\hline Statistic & Value (mean \pm SD) \\
\hline Intra-subject RSD (CV) & $20.3 \pm 10.1 \%$ \\
\hline Intra-measurement RSD (CV) & $29.6 \pm 9.6 \%$ \\
\hline Intra-subject RSE & $12.1 \pm 5.9 \%$ \\
\hline Intra-measurement RSE & $5.6 \pm 1.9 \%$ \\
\hline
\end{tabular}

RSD:- Relative standard deviation, CV: Coefficient of variation, RSE: Relative standard error

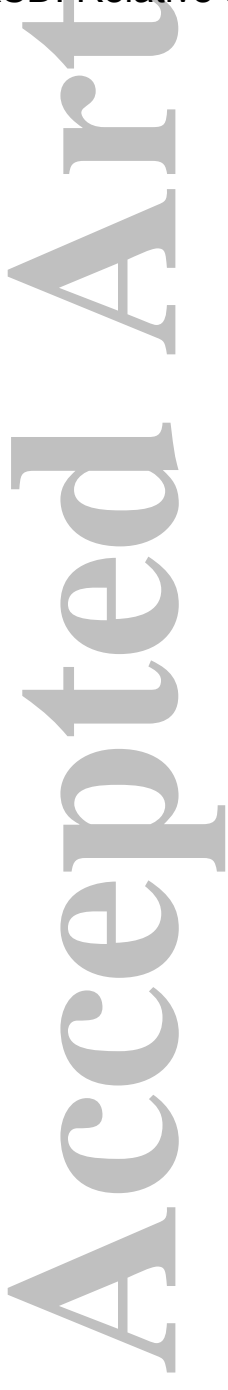


Table 2. Study Gestational Timelines for Preeclampsia Subjects

\begin{tabular}{|c|c|c|c|}
\hline Patient & Ultrasound Volume Flow Scan & Diagnosis (gestational age) & Delivery \\
\hline $1:$ & 29 weeks, 5 days & sPIH (34 weeks, 5 days) & 34 weeks, 5 days \\
\hline 16 & 32 weeks, 4 days & $\begin{array}{l}\text { mPIH (32 weeks, } 4 \text { days) } \\
\text { sPIH ( } 35 \text { weeks, } 1 \text { day) }\end{array}$ & 35 weeks, 1 day \\
\hline 18 & 34 weeks, 1 day & $\begin{array}{l}\text { gHTN ( } 31 \text { weeks, } 0 \text { days) } \\
\text { sPIH ( } 34 \text { weeks, } 4 \text { days) }\end{array}$ & 34 weeks, 4 days \\
\hline 19 & 33 weeks, 3 days & PIH (36 weeks, 5 days) & 36 weeks, 5 days \\
\hline 22 & 34 weeks, 2 days & gHTN (39 weeks, 0 days) & 39 weeks, 0 days \\
\hline
\end{tabular}

gHTN: Gestational hypertension, PIH: Pregnancy-induced hypertension [m: mild, s: severe]

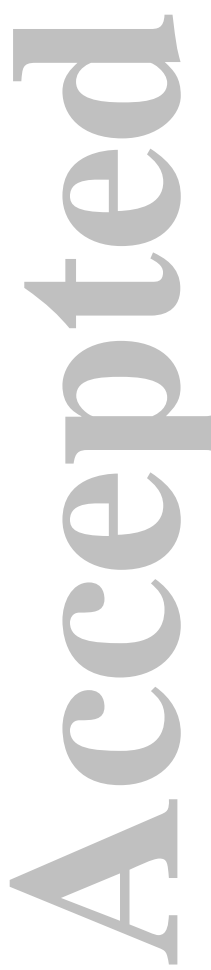




\section{Figure Captions}

Figure 1. Color flow image planes of the umbilical cord from an at-risk subject (gestation: 30 weeks and 3 days). (a) Axial-lateral and elevational-lateral (c-surface) views on the GE LOGIQ E9. Both views coincide at the marked center point (white dot) which also corresponds with the color flow focal depth of $8.86 \mathrm{~cm}$. The center point is positioned directly over the umbilical vein (blue) and two umbilical arteries (red) are shown surrounding the vein. (b) Elevational-lateral view from (a) shown in the offline analysis after segmenting the vein from the arteries and any adjacent vasculature and color flow artifacts. Color bars indicate velocity in centimeters per second.

Figure 2. Umbilical vein volume flow (absolute flow) as a function of gestational age for normal and preeclampsia subjects. Each data point is reported as the mean of a subject's three (nominal) free loop flow measurements. Error bars indicate standard error of the mean.

Figure 3. Umbilical vein volume flow (absolute flow) as a function of vessel imaging depth for normal and preeclampsia subjects. Each data point is reported as the mean of 30 (nominal) individual flow measurements at each free loop position. Error bars indicate standard error of the mean. A linear regression with $95 \%$ confidence interval $(\mathrm{Cl})$ is shown only for the normal group. Linear regression slopes $\left(95 \% \mathrm{Cl} ; \mathrm{r}^{2}\right)$ for the normal and preeclampsia groups are 22.73 (8.85 to $36.62 ; 0.31)$ and $21.16(7.02$ to $35.30 ; 0.53) \mathrm{mL} / \mathrm{min} / \mathrm{cm}$, respectively.

Figure 4. Comparisons of umbilical vein volume flow between normal $(\mathrm{N})$ and preeclampsia (PE) subjects based on (a) absolute flow, (b) depth-corrected flow, (c) weight-normalized flow, and (d) depth-corrected weight-normalized flow. Data points used in each group are the mean of a subject's three (nominal) free loop flow measurements. P values (two tailed) are an 
unpaired $t$ test of independent samples. Box-and-whisker plot: box extends from 25th to 75th percentile, whiskers extend from minimum to maximum values, line is median, and + is mean.

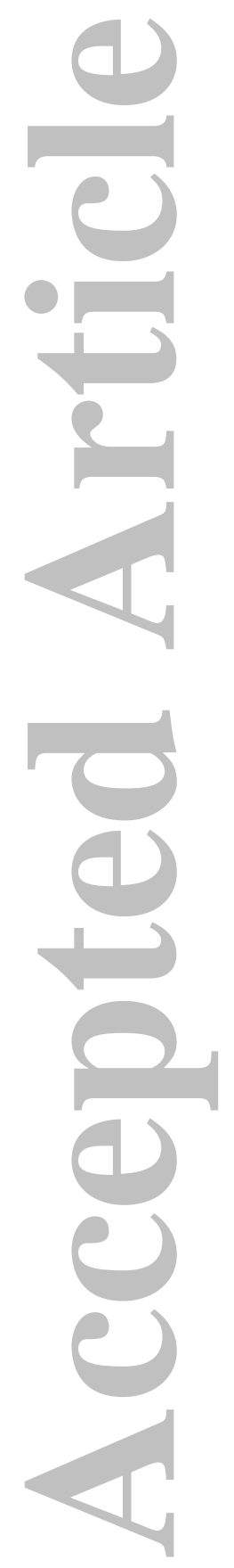



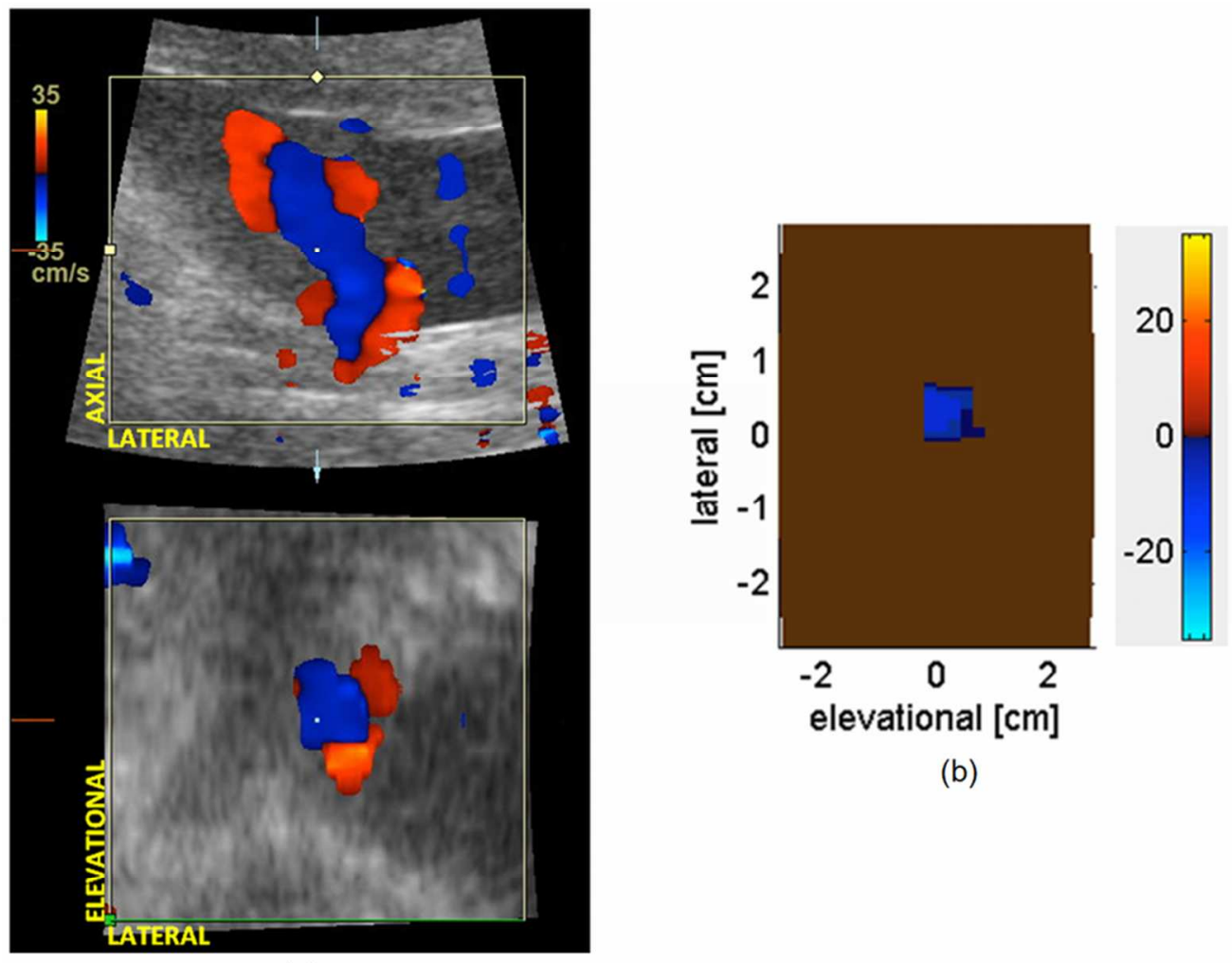

(a)

Figure 1. Color flow image planes of the umbilical cord from an at-risk subject (gestation: 30 weeks and 3 days). (a) Axial-lateral and elevational-lateral (c-surface) views on the GE LOGIQ E9. Both views coincide at the marked center point (white dot) which also corresponds with the color flow focal depth of $8.86 \mathrm{~cm}$. The center point is positioned directly over the umbilical vein (blue) and two umbilical arteries (red) are shown surrounding the vein. (b) Elevational-lateral view from (a) shown in the offline analysis after segmenting the vein from the arteries and any adjacent vasculature and color flow artifacts. Color bars indicate velocity in centimeters per second.

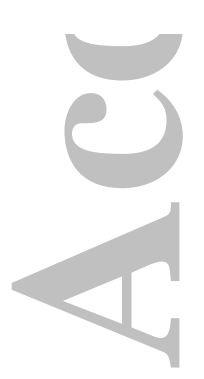

$40 \times 32 \mathrm{~mm}(600 \times 600$ DPI $)$ 


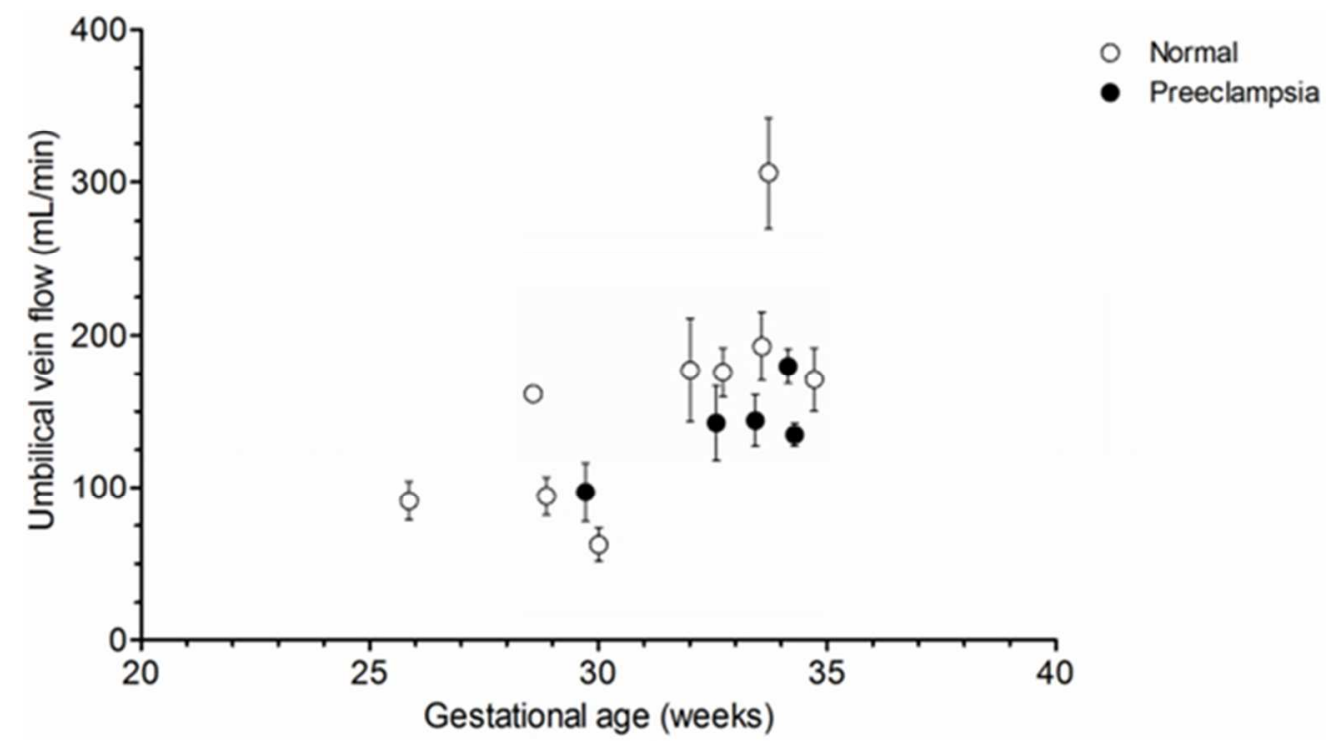

Figure 2. Umbilical vein volume flow (absolute flow) as a function of gestational age for normal and preeclampsia subjects. Each data point is reported as the mean of a subject's three (nominal) free loop flow measurements. Error bars indicate standard error of the mean.

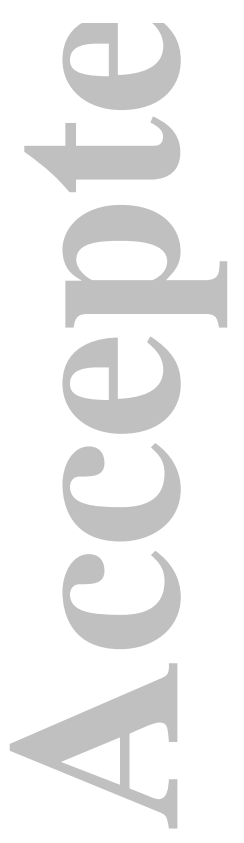

$27 \times 15 \mathrm{~mm}(600 \times 600$ DPI $)$ 


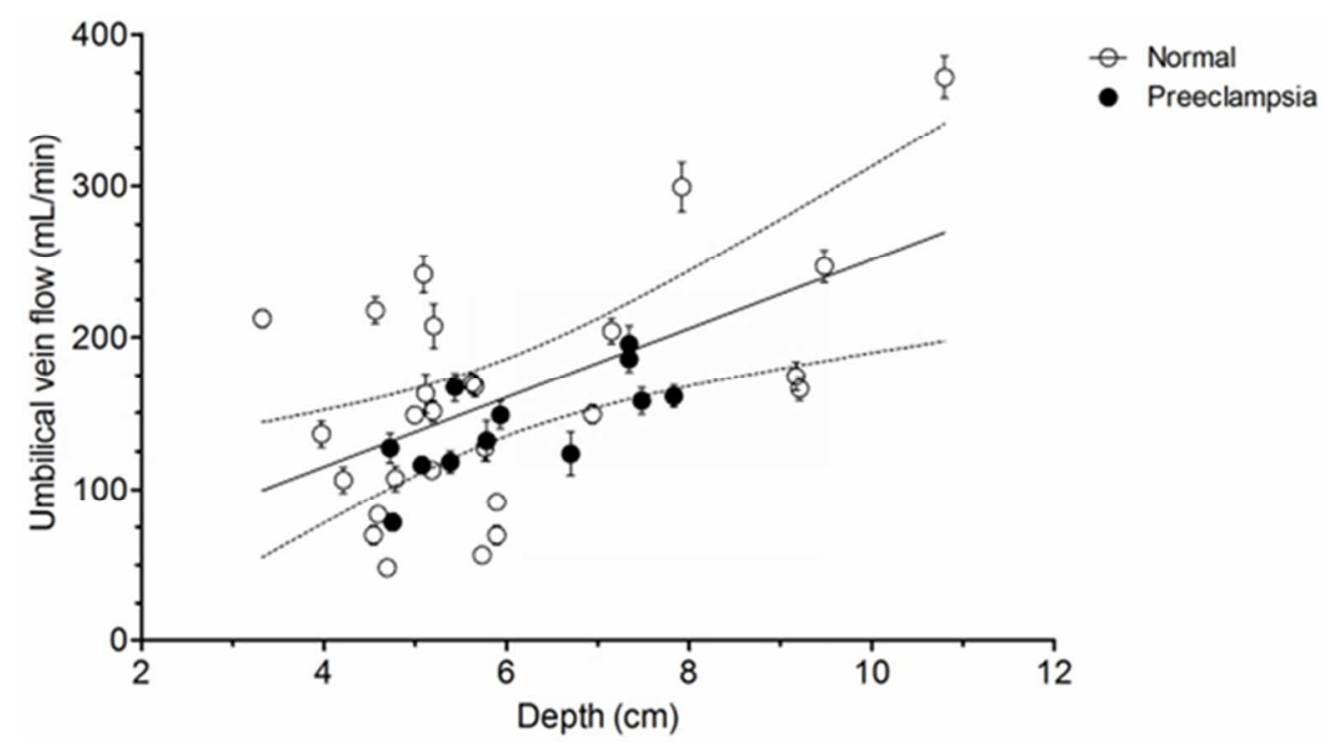

Figure 3. Umbilical vein volume flow (absolute flow) as a function of vessel imaging depth for normal and preeclampsia subjects. Each data point is reported as the mean of 30 (nominal) individual flow measurements at each free loop position. Error bars indicate standard error of the mean. A linear regression with $95 \%$ confidence interval (CI) is shown only for the normal group. Linear regression slopes (95\% CI; $r^{2}$ ) for the normal and preeclampsia groups are 22.73 (8.85 to $36.62 ; 0.31)$ and $21.16(7.02$ to $35.30 ; 0.53)$ $\mathrm{mL} / \mathrm{min} / \mathrm{cm}$, respectively.

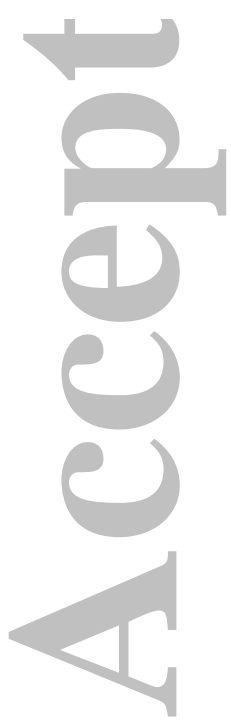

$27 \times 15 \mathrm{~mm}(600 \times 600 \mathrm{DPI})$ 


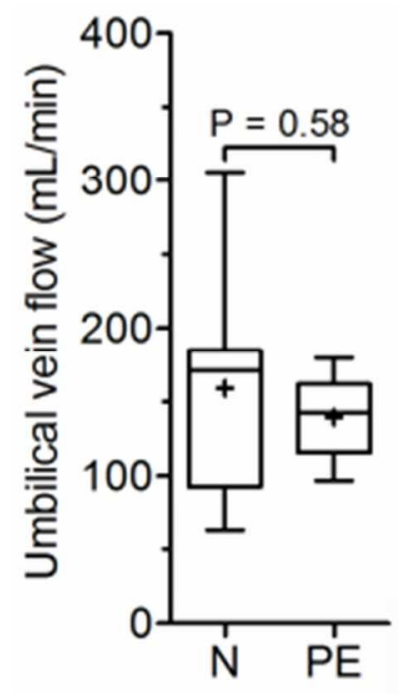

(a)

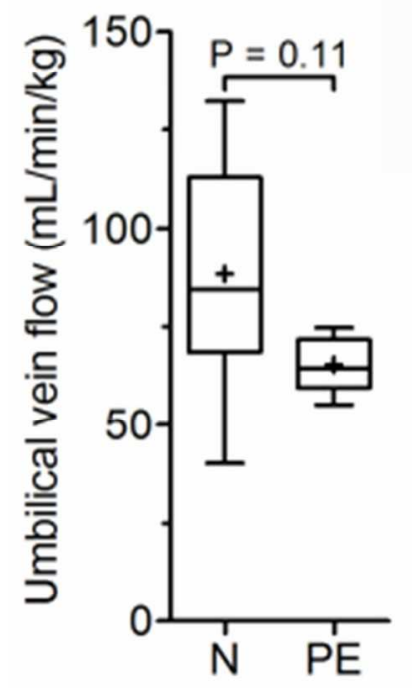

(c)

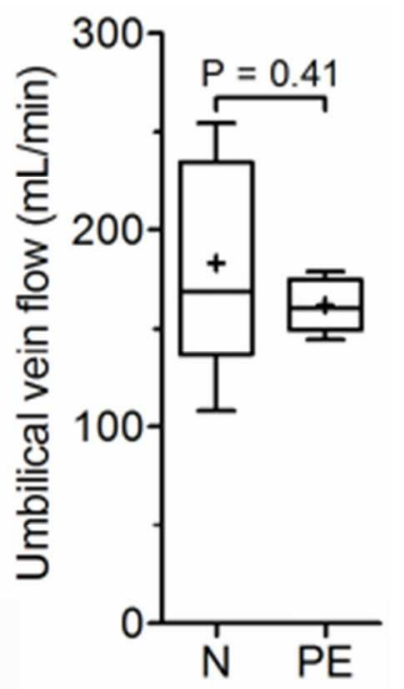

(b)

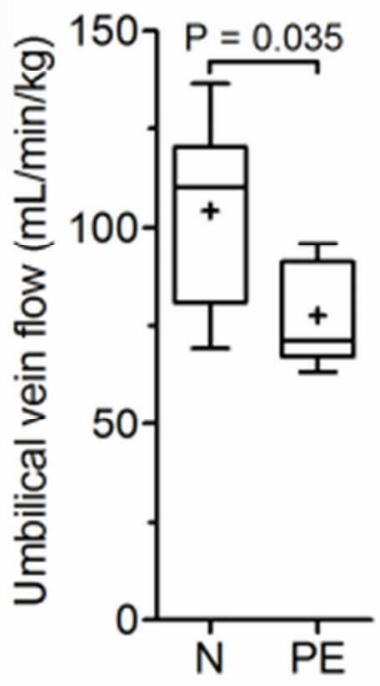

(d)

Figure 4. Comparisons of umbilical vein volume flow between normal $(N)$ and preeclampsia (PE) subjects based on (a) absolute flow, (b) depth-corrected flow, (c) weight-normalized flow, and (d) depth-corrected weight-normalized flow. Data points used in each group are the mean of a subject's three (nominal) free loop flow measurements. $P$ values (two tailed) are an unpaired $t$ test of independent samples. Box-andwhisker plot: box extends from 25th to 75th percentile, whiskers extend from minimum to maximum values, line is median, and + is mean.

$49 \times 79 \mathrm{~mm}(600 \times 600 \mathrm{DPI})$ 